$\xi_{p}$

\title{
Implementation of Geospatial Labeling using Time of Arrival and Cramer-Rao bound approach
}

\author{
M.Thanjaivadivel $* 1$, Ignatious K. Pious ${ }^{2}$ \\ ${ }^{12}$ Assistant Professor, Dept. of CSE, Veltech University, Avadi, Chennai \\ *Email: thanjaivadivel@gmail.com
}

\begin{abstract}
Internet of Things (IoT) is one of the most emerging technology worldwide and also plays pivotal role in sensing data and also provide communication between "things". In this paper, we implement calculation for geospatial labeling for Internet-of-Things (IoT) sort applications, which we indicate as location-of-things (LoT). The hidden thought of LoT applications is to utilize minimal effort of TWToA extending gadgets to perform restriction of labels. Two Way(TW) is a agreeable technique for deciding the range between two radio handset units. At the point when synchronization of the oscillators of the included transmitters is not reasonable, henceforth the tickers vary, at that point applying the estimation as a two courses go to the beneficiary and reflected back to the transmitter makes up for a portion of the stage contrasts between the oscillators included. We first propose TW-ToA localization algorithms may encounter execution debasement in situations where a portion of the APs are outside the correspondence scope of the labels. We at that point demonstrate that we can make utilization of the audible data (which demonstrates whether an AP is capable or unfit to speak with the labels). We also reformulate the restriction issue as a factual nonlinear estimation issue. To avoid ambiguity problem that arises only at few APs this has been sorted using Cramer-Rao bound approach.
\end{abstract}

Keywords: IOT, LOT, two-way time-of-arrival ranging TW-TOA, wireless sensor networks(WSN), localization, audibility.

\section{Introduction}

The issue of the indoor localization is tended to with systems of sensors taking extent based estimations within the sight of next to no earlier data. A few vigorous techniques are suggested that don't require past estimation crusades when a system is sent. The attention is on systems of ultra wideband sensors, however the proposed go based techniques can likewise be connected to different sorts of sensor systems. The area of an objective hub is evaluated from measured separations to stay hubs of known positions. The take into account the likelihood of extensive blunders in the range estimations because of UDP spread conditions. In relieving the UDP impact, the approach is to consolidate middle of the road area gauges from various subsets of guides. The novel criteria is proposed for distinguishing the blends that deliver awful gauges. These mixes are then disposed of in getting the last gauges. Reproductions uncover that the proposed strategies accomplish enhanced execution as for that of existing methods that adventure the same earlier data. Under numerous situations, the proposed techniques achieve the execution of a few calculations that adventure earlier data.

WASP Proposes to developed for high-accuracy localization and tracking. This platform uses the TOA of beacon signals periodically transmitted by the nodes at known times for localization. The system was designed to have a unique tradeoff between hardware complexity and processing complexity to provide high accuracy at minimal cost in complex radio propagation environments. To enable the system to perform well in realistic environments, it was also necessary to develop novel extensions to existing algorithms for the measurement of TOA, localization, and tracking. In this paper, we describe the architecture, hardware, and algorithms of WASP and present results based on field trials conducted in different radio propagation environments. The results show that WASP achieves a ranging accuracy of $0.15 \mathrm{~m}$ outdoors and $0.5 \mathrm{~m}$ indoors when around 12 anchor nodes are used. The accuracies are achieved with operating range of up to 200moutdoors and 30mindoors. This compares favorably to other published results for systems operating in realistic environments.

A typical procedure for aloof source localization is to use the range-contrast (RC) estimations between the source and a few spatially isolated sensors. The RC data characterizes an arrangement of hyperbolic conditions from which the source position can be figured with the information of the sensor positions. Under the standard presumption of Gaussian conveyed $\mathrm{RC}$ estimation blunders, it is notable that the greatest probability (ML) position estimation is accomplished by limiting a multimodal 
cost work which relates to a troublesome assignment. In correspondence to this, we propose to rough the non arched ML advancement by unwinding it to a curved enhancement issue utilizing semi distinct programming. A semi unmistakable unwinding RC-based situating calculation, which influences utilization of the acceptable source to position data, is proposed and its estimation execution is diverged from the two-advance weighted slightest squares technique and nonlinear minimum squares estimator and in addition Cramér- Rao bring down bound.

The quantity of gadgets on the Internet surpassed the quantity of individuals on the Internet in 2008, and is assessed to achieve 50 billion of every 2020. A colossal Internet of Things (IOT) biological community is developing to help the way toward associating genuine articles like structures, streets, family apparatuses, and human bodies to the Internet through sensors and microchip chips that record and transmit information, for example, sound waves, temperature, development, and different factors. The blast in Internet-associated sensors implies that new classes of specialized ability and application are being made. More granular every minute of every day measured observing is prompting a more profound comprehension of the inside and outside universes experienced by people. New information education practices, for example, relationship evaluation, abnormality location, and highrecurrence information handling are creating as people adjust to the various types of information streams empowered by the IOT. The IOT biological system has four basic practical advances: information creation, data era, which means making, and move making. This paper gives an exhaustive audit of the current and quickly developing environment of the Internet of Things (IOT).

The expression Internet of Things (IoT) messengers a dream without bounds Internet where associating physical things, from banknotes to bikes, through a system will give them a chance to take a dynamic part in the Internet, trading data about themselves and their environment. This will give quick access to data about the physical world and the items in it prompting creative administrations and increment in effectiveness and profitability. This paper examines the cutting edge of IoT and presents the key innovative drivers, potential applications, difficulties and future research regions in the area of IoT. IoT definitions from alternate point of view in scholarly and industry groups are additionally talked about and looked at. At long last some significant issues of future research in IoT are distinguished and talked about quickly.

\section{Disadvantages:}

1. The approach is less suitable for indoor environments.

2. The users in the LoT applications do not have access to Received Signal Strength measurements and is limited to using only TW-ToA measurements.

\section{Proposed System}

The proposed Grid-based localization system for tags, accurately calculate the locations through the help of Access Points and beacon packets.

Grid based localization approach first divides the map into grids. To evaluate the location of device, first marking algorithm is established, which calculates the possible grid positions of devices in a gridded map given the grid size. Then intersection points are noted, from the radius of its range. Based on the distance between devices the point in the grid the devices location is assumed.
The distance of the location is calculated based on time of arrival beacon packets from transmitting node.

\section{Advantages}

1.The approach provides considerable improvement over conventional methods by mitigating the well-known ambiguity problem which arises when only a few APs are audible.

2.It estimates the location of the tags.

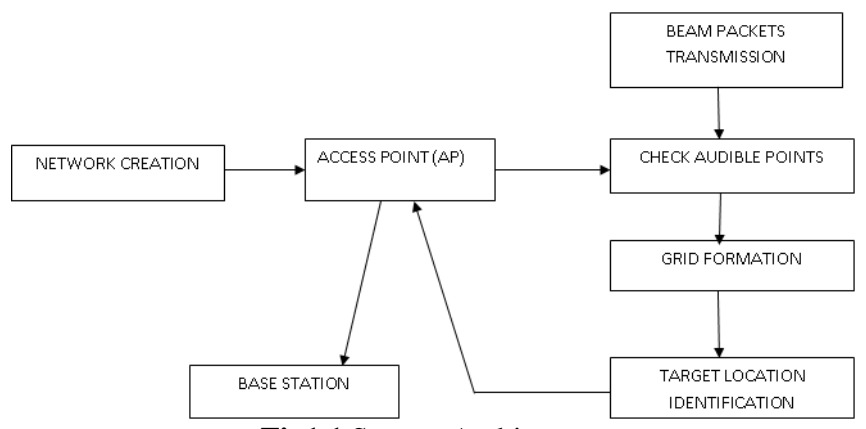

Fig1.1 System Architecture

\section{Design Modules}

\author{
1. Initialization \\ 2. Audibility Check \\ 3. Grid Formation \\ 4. Distance Calculation \\ 5. Location Identification
}

\section{Initialization}

The construction of the network with $\mathrm{N}$ Access Points whose locations are known and one target node with unknown location has been created. In the network area of 1600x900 dimension 20 nodes are deployed. Mesh topology has been formed among the nodes.

The use a circle shape and the black color to represents the nodes since its shape is most often associated with nodes in 2D plan. This helps to keep the viewer's attention, focus on the node and also help mimic an optical perspective of the network node. The nodes are allowed to move randomly. The colors used were maroon, blue, red and its complement green.

The red color denotes the target node. The color changes our logic is based on the event type. The three events occurring in the trace file are send ('s') for packets sent, receive ("r") for packets received. The node colors change according to the type of event that occurs at the node.

The blue color indicates hello message send and maroon color indicates receive hello packet.

\section{Audibility Check}

To check the audibility of the beacon packets which are transmitted to access points from target node and gets acknowledgement from access points, finds the Audible access points.

The access points which are in its communication range only can receive the beacon packet. The simulated results in the event are indicated by blue and maroon color. 
Access points which receives the beacon packet changes to maroon.Upon receiving the packet, access points send their location co-ordinate(x,y) message to target. Packet send time and receive time are identified from the trace file.

\section{- Grid Formation}

The network area is divided into grid

\section{- Distance Calculation}

The distance is calculated based on the send time and receive time interval. The distance between the devices are also calculated

\section{- Location Identification}

The intersection points of the grid lines are marked. The point closer to distance is chosen and calculated from above module. The identified location of the target is sent from the access point to the base station or severs.

\section{Result Analysis}

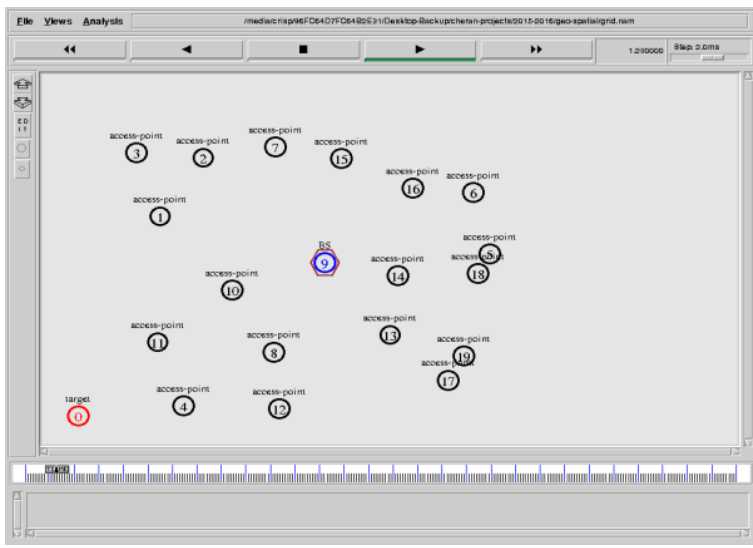

Fig 4.1 The formation of $n$ number of nodes to the base station is created using mesh topology.

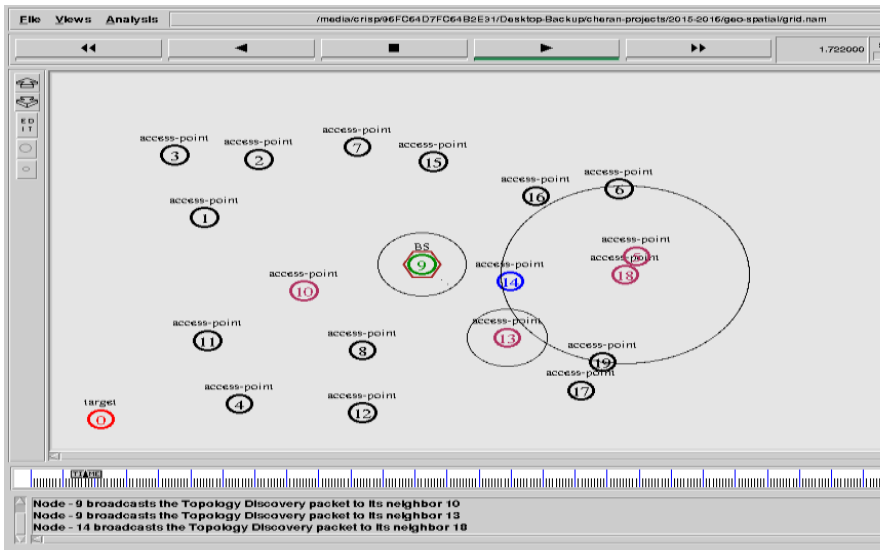

Fig 4.2 The base station tries to establish connection among the $n$ number of the APs.

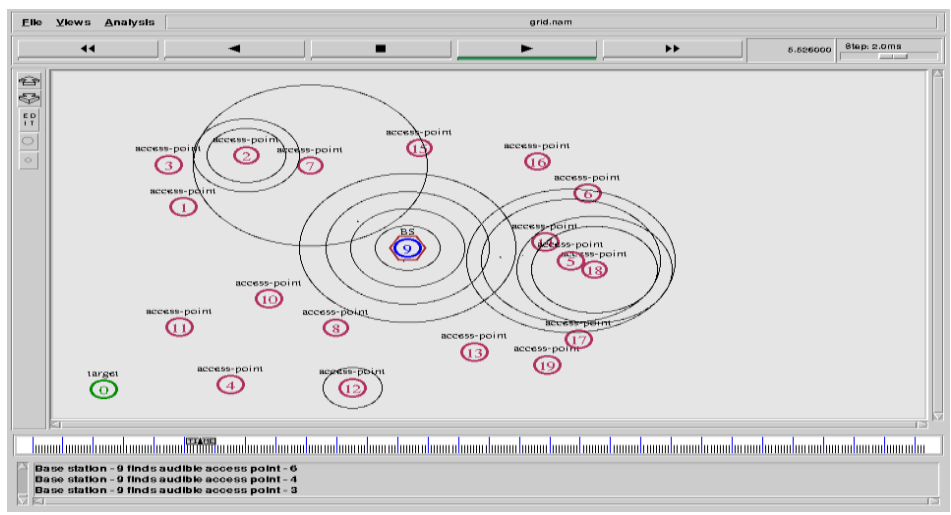

Fig 4.3 The connection establishment to the Access Point from the Base Station

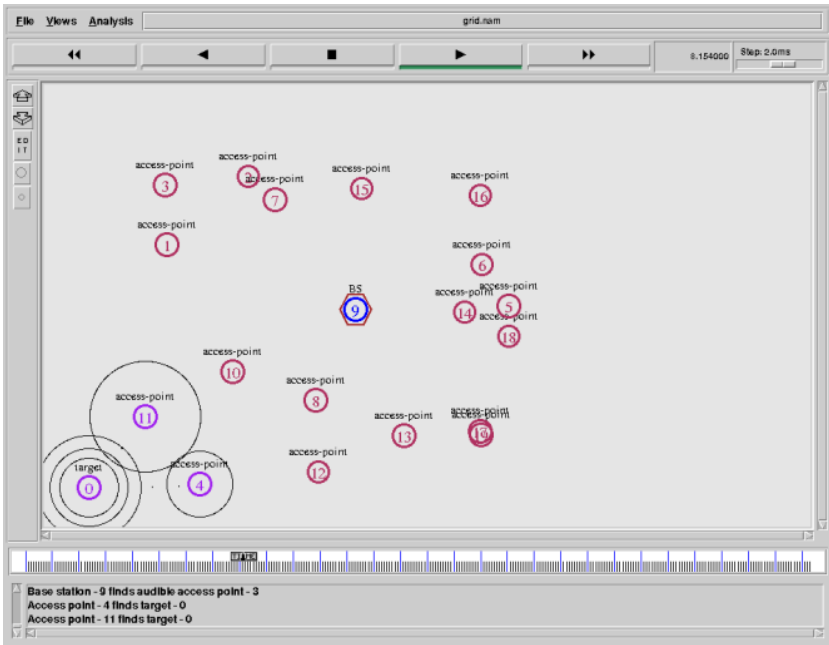

Fig 4.5 The one Access Point among those N number of the AP's near the Target node are set as temporary base station to identify the location of the Target node.

\section{Conclusion and Future Work}

Here we implement the calculation for Geospatial Tagging for Internet-of-Things (IoT) sort applications. The system for the proposed framework comprises of $n$ number of hubs and is made utilizing network topology. The server sends the signal parcels to recognize the accessible Access Points called Audible data.

In future the non capable of being heard node (target node) identified and the separation between those hubs are computed. The objective area is shared from the perceptible access focuses to the base station.

\section{References}

[1] E. Arias-de Reyna and P. M. Djuric, "Indoor localization with rangebased measurements and little prior information," IEEE Sensors J., vol. 13, no. 5, pp. 1979-1987, 2013. 
[2] G. Wang, S. Cai, Y. Li, and M. Jin, "Second-order cone relaxation for TOA-based source localization with unknown start transmission time," IEEE Trans. Veh. Technol., vol. 63, no. 6, pp. 2973-2977, Jul. 2014

[3] I. Chatzigiannakis, J. P. Drude, H. Hasemann, and A. Kröller, "Developing smart homes using the Internet of Things: How to demonstrate your system," in Distributed, Ambient, and Pervasive Interactions. New York, NY, USA: Springer, 2014, pp. 415-426.

[4] J. Gubbi, R. Buyya, S. Marusic, and M. Palaniswami, "Internet of things (IOT): A vision, architectural elements, and future directions," Future Gener. Comput. Syst., vol. 29, no. 7, pp. 1645-1660, 2013.

[5] N. Amiot, T. Pedersen, M. Laaraiedh, and B. Uguen, "A hybrid positioning method based on hypothesis testing," IEEE Lett. Wireless Commun., vol. 1, no. 4, pp. 348-351, Aug. 2012.

[6] W. R. Jung, S. Bell, A. Petrenko, and A. Sizo, "Potential risks of wifi-based indoor positioning and progress on improving localization functionality," in Proc. 4th ACM SIGSPATIAL Int. Workshop Indoor Spatial Awareness, 2012, pp. 13-20.

[7] Y. Wang, X. Ma, and G. Leus, "Robust time-based localization for asynchronous networks," IEEE Trans. Signal Process., vol. 59, no. 9, pp. 4397-4410, Sep. 2011.

[8] IEEE ISO/IEC 24730-5 information technology - Real-time locating systems (RTLS) - Part 5: Chirp spread spectrum (CSS) at $2.4 \mathrm{GHz}$ air interface, 2010 .

[9] (2015, Sep.). Timedomain, Timedomain Web-Site [Online]. Available: http://www.timedomain.com/

[10] K. W.-K. Lui, F. K. Chan, and H.-C. So, "Semidefinite programming approach for range-difference based source localization," IEEE Trans. Signal Process., vol. 57, no. 4, pp. 1630-1633, Apr. 2009. NEVAT et al.: LOCATION OF THINGS 185 